\title{
A Comparative Study of Successful and UnsuccessfulEFL Teachers in Terms of Performance and Teacher Interaction
}

\section{Zahra Zohoorian}

English Department, Mashhad Branch, Islamic Azad University, Mashhad, Iran Email: marjan.zohoorian@yahoo.com

\section{Akram Faravani*}

English Department, Mashhad Branch, Islamic Azad University, Mashhad, Iran afaravani@yahoo.com

\section{Abstract}

7 eachers have a focal role in every society and there is a bold emphasis on the need for successful teachers. In order to be a successful teacher one has to perform her/his knowledge well to have a good impact on students' learning. In addition, in order to enhance students' learning in class a teacher needs to communicate and interact well with them to create a good atmosphere for learning. Thus, two factors of performance and interaction can be leading factors in language teaching success. To find the Patterns of Teaching Performance and Teacher Interaction, the researchers sought participation of 50 English language teachers and their 200 English language learners in 20 English institutions. The instruments included successful teachers questionnaire, teaching performance questionnaire and teacher interaction questionnaire. After distinguishing the successful teachers, through running the descriptive statistics, it was found out that among the three sub-constructs of teaching performance, development and planning had the highest mean scores. Also, among the eight sub-constructs of teacher interaction, uncertain, leadership, and helpful and friendly behavior had the highest mean scores. Curriculum developers, policy makers and teacher trainers can include the instruction of such constructs in courses for novice teachers to help them on the way of success.

Key Words: Successful EFL Teachers, Teacher performance, Teacher Interaction 


\section{Introduction}

Teachers can rise the cultural and the economic characteristics of the educational society, and so make our young people ready to be effective citizens and independent lifelong learners, and this is crucial for their working opportunities. As Dubey (2016) mentioned the teachers' place in society is very important. A teacher can be considered as the axle for transferring intellectual traditions and technical skills from generation to generation and help to keep the civilization going.

Being a teacher does not only consist of being an assistant in the difficult activity of educating; it means being a creative and a highly talented guide, which follows a student through all the way of studying. Elliot (2015) claimed that teacher quality and performance are among the most important school variables that affect student achievement. As stated by Soares (2015), it is generally believed that good teaching consists of a good relation between the teacher and students and also among students. The best result in a classroom emerges from proper interaction between the teacher and the students. Therefore, the teachers' roles can be crucial to the effectiveness of the language learning. In addition, Whitaker (2004) mentioned that teachers should deeply believe that building relationships are imperative to the motivation process.

One critical factor for being a successful teacher, specially a successful language teacher is how the teacher performs. Montoneri (2011) stated that effective teaching performance is a vital factor in fostering students' learning improvement. Students' opinions about teachers at the end of each semester can indirectly provide useful information 
about teachers' performance. Choudhury (2005) considered interaction as an important element for language teachers. Zhi-Feng Liu, et al. (2013) have claimed that teacher and student interaction is proved to be an important concern in education, and teacher-student interaction is favorable in students' learning. For learning a foreign language, it is necessary to build mental, emotional and social interactions between teachers and students (Faramarz Zadeh, 2016; Faravani, 2021). Adaba (2017) claimed classroom interaction plays an important role in the process of language teaching and learning by giving learners opportunities to receive the input that is provided by the teacher, learners or material which must be understood by the learners in order to make them involved in the classroom task by providing the output. Interaction in English classroom is in the core of communication in this era of communicative language teaching. Therefore, teachers need to know to what extent their classroom practices facilitate language learning in reality. Eschenmann (2015) suggest that teachers have to build relationships to motivate their students to learn. Many studies have proposed that teachers have to believe that building relationships are important in the encouraging process (e.g. Eschenmann, 2015, Faravani \& Ataei, 2015a; Faravani \& Ataei, 2015b; Feng Liu, et al., 2013). Moreover, Fernández and Pérez Cañado (2001) state that teacher's performance can affect students' motivation and can make them interested in learning. Suwandee (1995) stated that there is a positive relationship between teachers' performance and teaching practices which in turn contribute to the development of teacher efficacy and can lead to the production of successful students. 
Effective teaching and its development is one of the main concerns for language teachers in the current era. Besides, in today's educational system and due to the changes which take place in this system, having professional and successful teachers to help students achieve their goals is necessary. Also, any big change in society necessities the growth of the educational system of that society which can be obtained by the improvement of teachers' performance. By keeping in mind the importance of teacher interaction and performance the theoretical framework of this study is based on mega planning. Mega planning starts with the question of "What kind of world do you want for your children?" Kaufman (2005) claimed that mega planning has an important role to define success and can identify the activities a person or a part of an organization should do to be successful. It views individuals and organizations as means to societal ends, and begins by identifying the results that an institution commits to contribute to society. Mega thinking and planning's first focus is on adding value for all stakeholders, including the shared society to define and get to persistent organizational success (Kaufman, 2009). While there has been extensive research on different factors affecting successful teaching, there is little research on teacher factors including performance and interaction among successful teachers. Thus, based on the objectives of the study, the following questions were answered.

1. What are the most important constructs of teaching performance among successful Iranian EFL teachers?

2. What are the most important constructs of teacher interaction among successful Iranian EFL teachers? 


\section{Methodology}

The present study relied on quantitative data from the survey. As the first stage the learners filled the successful teacher questionnaire based on which the 50 teachers were categorized in two groups of successful (high) and unsuccessful (low).

\section{Participants and Setting}

The target population for the present study included English teachers at institution level and also their students. To collect the required data 200 Iranian EFL language learners of 50 English teachers in advanced level of proficiency from 20 foreign language institutions of Mashhad, Iran, were selected through convenience sampling. The teachers were both male and female (23 male and 27 female). Their age ranged from 22 to 41 . Their students were both male and female, and their age ranged from 18 to 46 .

\subsection{Instrumentation}

To conduct this study, Successful English Teachers Questionnaire developed by Moafian and Pishghadam (2009) which includes 47 items on 5point Likert scale which defines 12 constructs of teaching accountability $(7$ items), interpersonal relationships(7items), attention to all(5 items), examination(3items), commitment(3items), learning boosters(4 items), creating a sense of competence(4 items), teaching boosters(4 items), physical and emotional acceptance( 2 items), empathy( 2 items), class attendance( 2 items) and dynamic(2 items) was employed. A reliability of 0.94 is reported by the developers. The 
second instrument was the final version of teaching Performance questionnaire developed by Moreno-Murcia, Torregrosa and Pedreno (2015). It consists of 28 items including the 3 constructs of planning (4 items), development (17 items), and result ( 7 items). The reliability of the questionnaire is reported by MorenoMurcia, Torregrosa and Pedreno (2015) as 0.94 for this questionnaire. The third instrument was the Questionnaire on Teacher Interaction (QTI) developed by Wubbels and Levy (1993) which has 48 items and employs a Likert scale. It define 8 constructs of leadership behaviour, understanding behaviour, uncertain behaviour, admonishing behaviour, helpful and friendly behaviour, student responsibility and freedom behaviour, dissatisfied behaviour and strict behaviour. The reliability is reported as follows for each of the constructs: leadership 0.83, helping/friendly 0.82, understanding 0.78, student responsibility/freedom 0.66 , uncertain 0.77 , dissatisfied 0.75 , admonishing 0.71 , and strict 0.63 .

\section{Results}

Teachers are divided into 2 groups based on their Teachers' success scores $(\mathrm{Norm}=81.31)$. The number of teachers in higher group (successful) was 29 and 21 in the lower group (unsuccessful). The results in table 1 show that in teacher interaction the highest mean score was for uncertain (Mean High=16.99) and the lowest mean score was for student responsibility and freedom (Mean High=9.95). In teaching performance the highest mean score was for development (Mean High=67.67), and the lowest mean score was for result (Mean Low=14.05). 
Table 1

Group Statistics for the 2 groups on teacher interaction and teaching performance

\begin{tabular}{|c|c|c|c|c|}
\hline & Group & $\mathbf{N}$ & Mean & Std. Deviation \\
\hline \multirow[t]{2}{*}{ Leadership } & High & 29 & 14.87 & 2.15 \\
\hline & Low & 21 & 13.69 & 1.95 \\
\hline \multirow[t]{2}{*}{ Understanding } & High & 29 & 12.13 & 2.22 \\
\hline & Low & 21 & 12.05 & 2.94 \\
\hline \multirow[t]{2}{*}{ Uncertain } & High & 29 & 16.99 & 1.82 \\
\hline & Low & 21 & 14.97 & 1.86 \\
\hline \multirow[t]{2}{*}{ Admonishing } & High & 29 & 10.74 & 3.02 \\
\hline & Low & 21 & 12.58 & 2.73 \\
\hline \multirow[t]{2}{*}{ Helpful/Friendly } & High & 29 & 15.04 & 1.68 \\
\hline & Low & 21 & 14.74 & 2.23 \\
\hline \multirow{2}{*}{$\begin{array}{c}\text { Student responsibility and } \\
\text { freedom }\end{array}$} & High & 29 & 9.95 & 2.53 \\
\hline & Low & 21 & 10.96 & 3.85 \\
\hline \multirow[t]{2}{*}{ Dissatisfied } & High & 29 & 13.31 & 2.22 \\
\hline & Low & 21 & 13.02 & 1.89 \\
\hline \multirow[t]{2}{*}{ Strict } & High & 29 & 11.08 & 2.37 \\
\hline & Low & 21 & 11.43 & 3.44 \\
\hline \multirow[t]{2}{*}{ Development } & High & 29 & 67.67 & 7.31 \\
\hline & Low & 21 & 62.44 & 4.78 \\
\hline \multirow[t]{2}{*}{ Planning } & High & 29 & 27.10 & 3.50 \\
\hline & Low & 21 & 24.74 & 2.82 \\
\hline \multirow[t]{2}{*}{ Result } & High & 29 & 15.17 & 2.26 \\
\hline & Low & 21 & 14.05 & $\mathbf{1 . 5 5}$ \\
\hline
\end{tabular}

To examine the differences between the 2 groups an independent sample t-test was performed. The results (Table 2) present that the 2 groups were significantly different in 3 (Leadership, $\mathrm{P}=.05, \mathrm{~T}=-1.98$; Uncertain, $\mathrm{P}=.00, \mathrm{~T}=-3.81$; and Admonishing $\mathrm{P}=.03, \mathrm{~T}=2.22$ ) out of 8 constructs of Teacher Interaction and in all the 3 constructs of Teaching Performance (Development, $\mathrm{P}=.00$, $\mathrm{T}=-2.85$; Planning, $\mathrm{P}=.01, \mathrm{~T}=-2.54$; Result, $\mathrm{P}=.05, \mathrm{~T}=-$ 1.96). 
Table 2

Independent Samples T-Test for the 2 groups on teacher interaction and teaching performance

\begin{tabular}{|c|c|c|c|c|c|c|}
\hline & \multirow{2}{*}{$\begin{array}{c}\text { Mean } \\
\text { Difference }\end{array}$} & \multirow[b]{2}{*}{$\mathbf{T}$} & \multirow{2}{*}{$\begin{array}{l}\text { Sig. (2- } \\
\text { tailed) }\end{array}$} & \multicolumn{2}{|c|}{$\begin{array}{l}95 \% \text { Confidence } \\
\text { Interval } \\
\end{array}$} \\
\hline & & & & & Lower & Upper \\
\hline \multirow[t]{2}{*}{ Leadership } & $\begin{array}{l}\text { Equal variances } \\
\text { assumed }\end{array}$ & -1.17 & $\begin{array}{c}-1.98 \\
\end{array}$ & .05 & -2.37 & .017 \\
\hline & $\begin{array}{l}\text { Equal variances } \\
\text { not assumed }\end{array}$ & -1.17 & -2.01 & .05 & -2.35 & .00 \\
\hline \multirow[t]{2}{*}{ Understanding } & $\begin{array}{c}\text { Equal variances } \\
\text { assumed }\end{array}$ & -.08 & -.11 & .91 & -1.54 & 1.38 \\
\hline & $\begin{array}{l}\text { Equal variances } \\
\text { not assumed }\end{array}$ & -.08 & -.10 & .91 & -1.63 & 1.46 \\
\hline \multirow[t]{2}{*}{ Uncertain } & $\begin{array}{c}\text { Equal variances } \\
\text { assumed }\end{array}$ & -2.01 & -3.81 & .00 & -3.08 & -.95 \\
\hline & $\begin{array}{l}\text { Equal variances } \\
\text { not assumed }\end{array}$ & -2.01 & -3.80 & .00 & -3.08 & -.94 \\
\hline \multirow[t]{2}{*}{ Admonishing } & $\begin{array}{c}\text { Equal variances } \\
\text { assumed }\end{array}$ & 1.84 & 2.22 & .03 & .17 & 3.52 \\
\hline & $\begin{array}{l}\text { Equal variances } \\
\text { not assumed }\end{array}$ & 1.84 & 2.25 & .02 & .20 & 3.49 \\
\hline \multirow[t]{2}{*}{$\begin{array}{c}\text { Helpful/Friendl } \\
y\end{array}$} & $\begin{array}{c}\text { Equal variances } \\
\text { assumed }\end{array}$ & -.29 & -.54 & .59 & -1.41 & .81 \\
\hline & $\begin{array}{l}\text { Equal variances } \\
\text { not assumed }\end{array}$ & -.29 & -.51 & .60 & -1.47 & .87 \\
\hline \multirow{2}{*}{$\begin{array}{c}\text { Student } \\
\text { responsibility } \\
\text { and freedom }\end{array}$} & $\begin{array}{c}\text { Equal variances } \\
\text { assumed }\end{array}$ & 1.00 & 1.11 & .26 & -.80 & 2.82 \\
\hline & $\begin{array}{l}\text { Equal variances } \\
\text { not assumed }\end{array}$ & 1.00 & 1.04 & .30 & -.95 & 2.96 \\
\hline \multirow[t]{2}{*}{ Dissatisfied } & $\begin{array}{c}\text { Equal variances } \\
\text { assumed }\end{array}$ & -.29 & $\begin{array}{l}.48 \\
\end{array}$ & .63 & $\begin{array}{l}-1.49 \\
\end{array}$ & .91 \\
\hline & $\begin{array}{l}\text { Equal variances } \\
\text { not assumed }\end{array}$ & -.29 & -.49 & .62 & -1.46 & .88 \\
\hline \multirow[t]{2}{*}{ Strict } & $\begin{array}{c}\text { Equal variances } \\
\text { assumed }\end{array}$ & .35 & .43 & .66 & -1.29 & 2.00 \\
\hline & $\begin{array}{l}\text { Equal variances } \\
\text { not assumed }\end{array}$ & .35 & .40 & .68 & -1.41 & 2.12 \\
\hline \multirow[t]{2}{*}{ Development } & $\begin{array}{c}\text { Equal variances } \\
\text { assumed }\end{array}$ & -5.22 & -2.85 & .00 & -8.90 & -1.55 \\
\hline & $\begin{array}{l}\text { Equal variances } \\
\text { not assumed }\end{array}$ & -5.22 & -3.05 & .00 & -8.67 & \begin{tabular}{ll|}
-1.78 \\
\end{tabular} \\
\hline \multirow[t]{2}{*}{ Planning } & $\begin{array}{c}\text { Equal variances } \\
\text { assumed }\end{array}$ & -2.35 & -2.54 & .01 & -4.22 & -.49 \\
\hline & $\begin{array}{l}\text { Equal variances } \\
\text { not assumed }\end{array}$ & -2.35 & -2.63 & .01 & -4.15 & -.55 \\
\hline \multirow[t]{2}{*}{ Result } & $\begin{array}{c}\text { Equal variances } \\
\text { assumed }\end{array}$ & -1.12 & -1.96 & .05 & -2.27 & .02 \\
\hline & $\begin{array}{l}\text { Equal variances } \\
\text { not assumed }\end{array}$ & -1.12 & -2.08 & .04 & -2.21 & -.03 \\
\hline
\end{tabular}


Concerning the constructs, in Leadership, the highest mean score was for item 5 (This teacher is a good leader.) and the lowest score was for item 4 (This teacher knows everything that goes on in the classroom.)

Results of the independent samples T-Test (table 4) showed that in Leadership, the 2 groups are significantly different in item 1 (This teacher talks enthusiastically about his/her subject), item 2 (This teacher explains things clearly), item 5 (This teacher is a good leader.) ( $\mathrm{P}=.00, \mathrm{~T}=-$ $3.62)$, and item 6 (This teacher acts confidently.) ( $\mathrm{P}=.05$, $\mathrm{T}=-2.00)$. In all the items teachers in higher group outperformed the lower one. However, in items 3 (This teacher holds our attention.) and 4 (This teacher knows everything that goes on in the classroom.) lower group received higher scores. (Though the differences are not significant).

\section{Table 4}

Independent Samples T- Test for Leadership

\begin{tabular}{|c|c|c|c|c|c|c|}
\hline & \multirow{2}{*}{$\begin{array}{c}\text { Mean } \\
\text { Difference } \\
-.38\end{array}$} & \multirow{2}{*}{$\begin{array}{l}\text { Sig. (2- } \\
\text { tailed) } \\
.03\end{array}$} & \multirow[b]{2}{*}{$T$} & \multicolumn{2}{|c|}{$\begin{array}{c}95 \% \\
\text { Confidence } \\
\text { Interval }\end{array}$} \\
\hline & & & & & $\begin{array}{l}\text { Lower } \\
-74\end{array}$ & \begin{tabular}{|l} 
Upper \\
-.02
\end{tabular} \\
\hline TIQ1 & Equal variances not &.- .38 & .03 & -2.20 & -.73 & -.02 \\
\hline \multirow[t]{2}{*}{ TIQ2 } & Equal variances assumed & -.68 & .00 & -4.53 & -.99 & -.38 \\
\hline & $\begin{array}{l}\text { Equal variances not } \\
\text { assumed }\end{array}$ & -.68 & .00 & -4.45 & -.99 & -.37 \\
\hline \multirow[t]{2}{*}{ TIQ3 } & Equal variances assumed & .38 & .14 & 1.49 & -.13 & .90 \\
\hline & $\begin{array}{c}\text { Equal variances not } \\
\text { assumed }\end{array}$ & .38 & .14 & 1.47 & -.14 & .90 \\
\hline \multirow[t]{2}{*}{ TIQ4 } & Equal variances assumed & .38 & .13 & 1.51 & -.12 & .89 \\
\hline & $\begin{array}{l}\text { Equal variances not } \\
\text { assumed }\end{array}$ & .38 & .14 & 1.47 & -.14 & .90 \\
\hline \multirow[t]{2}{*}{ TIQ5 } & Equal variances assumed & -.50 & .00 & -3.62 & -.79 & -.22 \\
\hline & $\begin{array}{c}\text { Equal variances not } \\
\text { assumed }\end{array}$ & -.50 & .00 & -3.62 & -.79 & -.22 \\
\hline \multirow[t]{2}{*}{ TIQ6 } & Equal variances assumed & -.36 & .05 & -2.00 & -.73 & .00 \\
\hline & $\begin{array}{c}\text { Equal variances not } \\
\text { assumed }\end{array}$ & -.36 & .04 & -2.06 & -.72 & -.01 \\
\hline
\end{tabular}


In Understanding construct (table 5) shows that the highest mean score was for item 9 (This teacher is willing to explain things again.) (Mean Low $=3.78, \mathrm{SD}=.63$ ), and the lowest score was for item 8 (If we don't agree with this teacher, we can talk about it.) (Mean High= .70, $\mathrm{SD}=.72$ ).

Table 5

Group Statistics for understanding construct

\begin{tabular}{|c|c|c|c|c|}
\hline & Group & $\mathbf{N}$ & Mean & Std. Deviation \\
\hline \multirow[t]{2}{*}{ TIQ7 } & High & 29 & 1.02 & .74 \\
\hline & Low & 21 & 1.30 & .96 \\
\hline \multirow[t]{2}{*}{ TIQ8 } & High & 29 & .70 & .72 \\
\hline & Low & 21 & 1.14 & .97 \\
\hline \multirow[t]{2}{*}{ TIQ9 } & High & 29 & 3.27 & .62 \\
\hline & Low & 21 & 3.78 & .63 \\
\hline \multirow[t]{2}{*}{ TIQ10 } & High & 29 & 3.21 & .56 \\
\hline & Low & 21 & 2.53 & .49 \\
\hline \multirow[t]{2}{*}{ TIQ11 } & High & 29 & 1.21 & 1.03 \\
\hline & Low & 21 & 1.76 & 1.12 \\
\hline \multirow[t]{2}{*}{ TIQ12 } & High & 29 & 2.72 & .79 \\
\hline & Low & 21 & 2.53 & .47 \\
\hline
\end{tabular}

Table 6

Independent Samples T-Test for Understanding Construct

\begin{tabular}{|c|c|c|c|c|c|c|}
\hline & \multirow{2}{*}{$\begin{array}{l}\text { Mean } \\
\text { Differen } \\
\text { e }\end{array}$} & \multirow[b]{2}{*}{$\mathbf{T}$} & \multirow{2}{*}{$\begin{array}{c}\text { Sig. } \\
(2- \\
\text { tailed }\end{array}$} & \multicolumn{2}{|c|}{$\begin{array}{c}95 \% \\
\text { Confidenc } \\
\text { Interval } \\
\text { Lowe } \mid \text { Upp }\end{array}$} \\
\hline & & & & & & \\
\hline \multirow[t]{2}{*}{ TIQ7 } & Equal variances assumed & .28 & 1.18 & .24 & -.20 & \\
\hline & Equal variances not assumed & .28 & 1.13 & .26 & -.22 & \\
\hline \multirow[t]{2}{*}{ TIQ8 } & Equal variances assumed & .44 & 1.83 & .07 & -.04 & \\
\hline & Equal & .44 & 1.74 & .09 & -.07 & \\
\hline \multirow[t]{2}{*}{ TIQ9 } & Equa & -.48 & -2.71 & .00 & -.85 & \\
\hline & Equal & -.48 & -2.70 & .01 & -.85 & \\
\hline TIQ1 & Equa & -.67 & -4.37 & .00 & -.98 & \\
\hline & Equal & -.67 & -4.47 & .00 & -.97 & \\
\hline & Equal & .54 & 1.76 & .08 & -.07 & \\
\hline & Equal va & .54 & 1.74 & .08 & -.08 & \\
\hline & & -.18 & -.95 & .34 & -.58 & \\
\hline & sumed & -.18 & -1.03 & .30 &. .55 & \\
\hline
\end{tabular}


The results of the independent samples T-Test in table 6 shows that the 2 groups are significantly different in item 9 (This teacher willing to explain things again.) ( $\mathrm{P}=.00$, $\mathrm{T}=-2.71$ ), and item 10 (If we have something to say, this teacher will listen.) $(\mathrm{P}=.00, \mathrm{~T}=-4.37)$. However, the differences in the other items were not significant.

In Uncertain construct, results (table 7) show that, the highest mean score was for item 14 (This teacher is hesitant.) (Mean High= 3.41, $\mathrm{SD}=.49$ ), and the lowest score was for item 16 (This teacher lets us boss her/him around.) (Mean High= 1.15, $\mathrm{SD}=1.02$ ).

Table 7

Group Statistics for Uncertain Construct

\begin{tabular}{|c|c|c|c|c|}
\hline & Group & $\mathbf{N}$ & Mean & Std. Deviation \\
\hline \multirow[t]{2}{*}{ TIQ13 } & High & 29 & 3.23 & .64 \\
\hline & Low & 21 & 2.62 & .50 \\
\hline \multirow[t]{2}{*}{ TIQ14 } & High & 29 & 3.41 & .49 \\
\hline & Low & 21 & 3.02 & .60 \\
\hline \multirow[t]{2}{*}{ TIQ15 } & High & 29 & 2.68 & .67 \\
\hline & Low & 21 & 2.20 & .75 \\
\hline \multirow[t]{2}{*}{ TIQ16 } & High & 29 & 1.15 & 1.02 \\
\hline & Low & 21 & 1.42 & 1.13 \\
\hline \multirow[t]{2}{*}{ TIQ17 } & High & 29 & 3.38 & .50 \\
\hline & Low & 21 & 3.05 & .55 \\
\hline \multirow[t]{2}{*}{ TIQ18 } & High & 29 & 3.15 & .61 \\
\hline & Low & 21 & 2.67 & .60 \\
\hline
\end{tabular}

An independent samples T-Test was run for the two groups for the uncertain construct.

The results of the independent samples T-Test (table 8) show that, the 2 groups were significantly different in item 13 (This teacher seems uncertain.) $(\mathrm{P}=.00, \mathrm{~T}=-3.66)$, item 14 (This teacher is hesitant.) $(\mathrm{P}=.01, \mathrm{~T}=-2.49)$, item 15 (This teacher acts as if he/she does not know what to do.) 
$(\mathrm{P}=.02, \mathrm{~T}=-2.36$ ), item 17 (This teacher is not sure what to do when we fool around.) $(\mathrm{P}=.03, \mathrm{~T}=-2.18)$, and item 18 (It's easy to make a fool out of this teacher.) $(\mathrm{P}=.00, \mathrm{~T}=-$ 2.72). In all of them teachers in higher group outperformed the lower one. However, in TIQ16 (This teacher lets us boss her/him around.) higher group teachers received the lower scores. (Though the difference is not significant).

Table 8

Independent Samples T-Test for Uncertain Construct

\begin{tabular}{|c|c|c|c|c|c|c|}
\hline & \multirow{2}{*}{$\begin{array}{c}\text { Mean } \\
\text { Difference }\end{array}$} & \multirow[b]{2}{*}{$\mathbf{T}$} & \multirow{2}{*}{$\begin{array}{l}\text { Sig. (2- } \\
\text { tailed) }\end{array}$} & \multicolumn{2}{|c|}{$\begin{array}{l}\text { 95\% Confidence } \\
\text { Interval }\end{array}$} \\
\hline & & & & & Lower & Upper \\
\hline \multirow[t]{2}{*}{ TIQ13 } & $\begin{array}{c}\text { Equal variances } \\
\text { assumed }\end{array}$ & -.61 & -3.66 & .00 & -.95 & -.27 \\
\hline & $\begin{array}{c}\text { Equal variances not } \\
\text { assumed }\end{array}$ & -.61 & -3.80 & .00 & -.94 & -.29 \\
\hline \multirow[t]{2}{*}{ TIQ14 } & \begin{tabular}{|c|}
$\begin{array}{c}\text { Equal variances } \\
\text { assumed }\end{array}$ \\
\end{tabular} & -.38 & -2.49 & .01 & -.70 & -.07 \\
\hline & $\begin{array}{c}\text { Equal variances not } \\
\text { assumed }\end{array}$ & -.38 & -2.41 & .02 & $\begin{array}{l}-.71 \\
\end{array}$ & -.06 \\
\hline \multirow[t]{2}{*}{ TIQ15 } & \begin{tabular}{|c|}
$\begin{array}{c}\text { Equal variances } \\
\text { assumed }\end{array}$ \\
\end{tabular} & -.47 & -2.36 & .02 & -.88 & -.07 \\
\hline & $\begin{array}{c}\text { Equal variances not } \\
\text { assumed }\end{array}$ & -.47 & -2.32 & .02 & -.89 & -.06 \\
\hline \multirow[t]{2}{*}{ TIQ16 } & $\begin{array}{c}\text { Equal variances } \\
\text { assumed }\end{array}$ & .27 & .88 & .38 & -.34 & .89 \\
\hline & $\begin{array}{c}\text { Equal variances not } \\
\text { assumed }\end{array}$ & .27 & .87 & .38 & -.35 & .90 \\
\hline \multirow[t]{2}{*}{ TIQ17 } & $\begin{array}{c}\text { Equal variances } \\
\text { assumed }\end{array}$ & -.32 & -2.18 & .03 & -.63 & -.02 \\
\hline & $\begin{array}{c}\text { Equal variances not } \\
\text { assumed }\end{array}$ & -.32 & -2.14 & .03 & -.63 & -.02 \\
\hline \multirow[t]{2}{*}{ TIQ18 } & $\begin{array}{c}\text { Equal variances } \\
\text { assumed }\end{array}$ & -.47 & -2.72 & .00 & -.82 & -.12 \\
\hline & $\begin{array}{c}\text { Equal variances not } \\
\text { assumed }\end{array}$ & -.47 & -2.73 & .00 & -.82 & -.12 \\
\hline
\end{tabular}

In Admonishing construct the results (table 9) show that, the highest mean score was for item 21 (This teacher is too quick to correct us when we break a rule.) (Mean 
High=3.40, SD= .67), and the lowest score was for item 20 (This teacher gets angry quickly.) (Mean High $=.86, \mathrm{SD}=$ $.89)$.

Table 9

Group Statistics for Admonishing Construct

\begin{tabular}{|c|c|c|c|c|}
\hline & Group & N & Mean & Std. Deviation \\
\hline TIQ19 & High & 29 & .94 & .99 \\
\hline & Low & 21 & 1.54 & .76 \\
\hline TIQ20 & High & 29 & .86 & .89 \\
\hline & Low & 21 & 1.61 & .73 \\
\hline TIQ21 & High & 29 & 3.40 & .67 \\
\hline & Low & 21 & 3.03 & .38 \\
\hline TIQ23 & High & 29 & 1.18 & .77 \\
\hline & Low & 21 & 1.75 & .89 \\
\hline TIQ24 & High & 29 & 1.07 & .80 \\
\hline & Low & 21 & 1.82 & .91 \\
\hline
\end{tabular}

The results of the independent samples T-Test (table 10) show that, the 2 groups were significantly different in item 19 (This teacher gets angry unexpectedly.) $(\mathrm{P}=.02$, $\mathrm{T}=2.29)$, item 20 (This teacher gets angry quickly.) ( $\mathrm{P}=.00$, $\mathrm{T}=3.15$ ), item 21 (This teacher is too quick to correct us when we break a rule.) $(\mathrm{P}=.02, \mathrm{~T}=-2.25)$, item 23 (It is easy to pick a fight with this teacher. $)(\mathrm{P}=.02, \mathrm{~T}=2.32)$ and item 24 (This teacher is sarcastic.) $(\mathrm{P}=.00, \mathrm{~T}=2.83)$. But, in item 22 (This teacher is impatient.) the difference was not significant. 
JRCIET

Vol. 7, No. 4

Dctober 2021

Table 10

Independent Samples T- Test for Admonishing Construct

\begin{tabular}{|c|c|c|c|c|c|c|}
\hline & & & & & $\begin{array}{c}95 \% \\
\text { confidenc } \\
\text { e Interval }\end{array}$ & \\
\hline & & $\begin{array}{c}\text { Mean } \\
\text { Differen } \\
\text { ce }\end{array}$ & $\mathbf{T}$ & $\begin{array}{c}\text { Sig. } \\
(2- \\
\text { tailed })\end{array}$ & Lower & Upper \\
\hline \multirow[t]{2}{*}{ TIQ19 } & $\begin{array}{c}\text { Equal } \\
\text { variances } \\
\text { assumed }\end{array}$ & .59 & 2.29 & .02 & .07 & 1.12 \\
\hline & $\begin{array}{c}\text { Equal } \\
\text { variances not } \\
\text { assumed }\end{array}$ & .59 & 2.39 & .02 & .09 & 1.09 \\
\hline \multirow[t]{2}{*}{ TIQ20 } & $\begin{array}{c}\text { Equal } \\
\text { variances } \\
\text { assumed }\end{array}$ & .75 & 3.15 & .00 & .27 & 1.23 \\
\hline & $\begin{array}{c}\text { Equal } \\
\text { variances not } \\
\text { assumed }\end{array}$ & .75 & 3.25 & .00 & .28 & 1.21 \\
\hline \multirow[t]{2}{*}{ TIQ21 } & $\begin{array}{c}\text { Equal } \\
\text { variances } \\
\text { assumed }\end{array}$ & -.37 & $\overline{2.25}$ & .02 & -.70 & -.04 \\
\hline & $\begin{array}{c}\text { Equal } \\
\text { variances not } \\
\text { assumed }\end{array}$ & -.37 & $\overline{2.45}$ & .01 & -.67 & -.06 \\
\hline \multirow[t]{2}{*}{ TIQ22 } & $\begin{array}{c}\text { Equal } \\
\text { variances } \\
\text { assumed }\end{array}$ & -.44 & $\overline{1.91}$ & .06 & -.91 & .02 \\
\hline & $\begin{array}{c}\text { Equal } \\
\text { variances not } \\
\text { assumed }\end{array}$ & -.44 & $\overline{1.88}$ & .06 & -.92 & .03 \\
\hline \multirow[t]{2}{*}{ TIQ23 } & $\begin{array}{c}\text { Equal } \\
\text { variances } \\
\text { assumed } \\
\end{array}$ & .57 & 2.32 & .02 & .07 & 1.06 \\
\hline & $\begin{array}{c}\text { Equal } \\
\text { variances not } \\
\text { assumed }\end{array}$ & .57 & 2.36 & .02 & .08 & 1.05 \\
\hline \multirow[t]{2}{*}{ TIQ24 } & $\begin{array}{c}\text { Equal } \\
\text { variances } \\
\text { assumed }\end{array}$ & .74 & 2.83 & .00 & .21 & 1.27 \\
\hline & $\begin{array}{c}\text { Equal } \\
\text { variances not } \\
\text { assumed }\end{array}$ & .74 & 2.83 & .00 & .21 & 1.27 \\
\hline
\end{tabular}


In Helpful/Friendly construct (table 11), the results show that the highest mean score was for item 29 (This teacher can take a joke.) (Mean High=3.49, $\mathrm{SD}=.57$ ), and the lowest score was for item 27 (This teacher is someone we can depend on.) (Mean High=1.16, $\mathrm{SD}=1.01)$.

Table 11

Group Statistics for Helpful/Friendly Construct

\begin{tabular}{|c|c|c|c|c|}
\hline & Group & N & Mean & Std. Deviation \\
\hline TIQ25 & High & 29 & 3.28 & .66 \\
\hline TIQ26 & High & 29 & 2.72 & .76 \\
\hline & Low & 21 & 2.60 & .51 \\
\hline TIQ27 & High & 29 & 1.16 & 1.01 \\
\hline & Low & 21 & 1.82 & 1.01 \\
\hline TIQ28 & High & 29 & 2.10 & .89 \\
\hline & Low & 21 & 2.12 & .68 \\
\hline TIQ29 & High & 29 & 3.49 & .57 \\
\hline & Low & 21 & 2.88 & .67 \\
\hline TIQ30 & High & 29 & 2.30 & .78 \\
\hline & Low & 21 & 2.43 & .59 \\
\hline
\end{tabular}

The results of the independent samples T-Test in table 12 show that, the 2 groups are significantly different in item 25 (This teacher helps us with our work.) ( $\mathrm{P}=.02, \mathrm{~T}=-$ 2.39), item 27 (This teacher is someone we can depend on.) $(\mathrm{P}=.02, \mathrm{~T}=2.62)$, and item 29 (This teacher can take a joke. $(\mathrm{P}=.00, \mathrm{~T}=-3.43)$. 
Table 12

Independent Samples T-Test for Helpful/Friendly Construct

\begin{tabular}{|c|c|c|c|c|c|c|}
\hline & & & & & $\begin{array}{c}95 \% \\
\text { Confide } \\
\text { nce } \\
\text { Interval }\end{array}$ & \\
\hline & & $\begin{array}{c}\text { Mean } \\
\text { Differen } \\
\text { ce }\end{array}$ & $\mathbf{T}$ & $\begin{array}{c}\text { Sig. } \\
(2- \\
\text { tailed })\end{array}$ & Lower & $\begin{array}{c}\text { Upp } \\
\text { er }\end{array}$ \\
\hline \multirow[t]{2}{*}{$\begin{array}{c}\text { TIQ2 } \\
5\end{array}$} & $\begin{array}{c}\text { Equal } \\
\text { variances } \\
\text { assumed }\end{array}$ & -.40 & $2 . \overline{39}$ & .02 & -.73 & -.06 \\
\hline & $\begin{array}{c}\text { Equal } \\
\text { variances not } \\
\text { assumed }\end{array}$ & -.40 & $2 . \overline{54}$ & .01 & -.71 & -.08 \\
\hline \multirow[t]{2}{*}{$\begin{array}{c}\text { TIQ2 } \\
6\end{array}$} & $\begin{array}{c}\text { Equal } \\
\text { variances } \\
\text { assumed }\end{array}$ & -.11 & -.57 & .56 & -.49 & .27 \\
\hline & $\begin{array}{c}\text { Equal } \\
\text { variances not } \\
\text { assumed }\end{array}$ & -.11 & -.61 & .54 & -.47 & .25 \\
\hline \multirow[t]{2}{*}{$\begin{array}{c}\text { TIQ2 } \\
7\end{array}$} & $\begin{array}{c}\text { Equal } \\
\text { variances } \\
\text { assumed }\end{array}$ & .66 & 2.26 & .02 & .07 & 1.24 \\
\hline & $\begin{array}{c}\text { Equal } \\
\text { variances not } \\
\text { assumed }\end{array}$ & .66 & 2.70 & .02 & .07 & 1.24 \\
\hline \multirow[t]{2}{*}{$\begin{array}{c}\text { TIQ2 } \\
8\end{array}$} & $\begin{array}{c}\text { Equal } \\
\text { variances } \\
\text { assumed }\end{array}$ & .02 & .08 & .93 & -.45 & .49 \\
\hline & $\begin{array}{c}\text { Equal } \\
\text { variances not } \\
\text { assumed }\end{array}$ & .02 & .08 & .92 & -.42 & .46 \\
\hline \multirow[t]{2}{*}{$\begin{array}{c}\text { TIQ2 } \\
9\end{array}$} & $\begin{array}{c}\text { Equal } \\
\text { variances } \\
\text { assumed }\end{array}$ & -.60 & $\overline{3.43}$ & .00 & -.96 & -.25 \\
\hline & $\begin{array}{c}\text { Equal } \\
\text { variances not } \\
\text { assumed }\end{array}$ & -.60 & $\overline{3.33}$ & .00 & -.97 & -.23 \\
\hline \multirow[t]{2}{*}{$\begin{array}{c}\text { TIQ3 } \\
\text { 0 }\end{array}$} & $\begin{array}{c}\text { Equal } \\
\text { variances } \\
\text { assumed } \\
\end{array}$ & .13 & .66 & .50 & -.27 & .54 \\
\hline & $\begin{array}{c}\text { Equal } \\
\text { variances not } \\
\text { assumed }\end{array}$ & .13 & .69 & .49 & -.25 & .52 \\
\hline
\end{tabular}


In Student responsibility and freedom construct the results in table 13 show that, the highest mean score was for item 36 (This teacher is lenient.) (Mean High= 2.39, $\mathrm{SD}=.70$ ), and the lowest score was for item 35 (This teacher give us a lot of free time in class.) (Mean High= $.62, \mathrm{SD}=.74)$.

Table 13

Group Statistics for Student responsibility and freedom construct

\begin{tabular}{|c|c|c|c|c|}
\hline & Group & N & Mean & Std. Deviation \\
\hline TIQ31 & High & 29 & .87 & .67 \\
\hline & Low & 21 & 1.48 & .86 \\
\hline TIQ32 & High & 29 & 1.99 & .98 \\
\hline & Low & 21 & 1.68 & .87 \\
\hline TIQ33 & High & 29 & 2.29 & 1.01 \\
\hline & Low & $\mathbf{2 1}$ & $\mathbf{2 . 3 8}$ & .71 \\
\hline TIQ34 & High & 29 & 1.79 & 1.13 \\
\hline & Low & 21 & 1.65 & .83 \\
\hline TIQ35 & High & 29 & .62 & .74 \\
\hline & Low & 21 & 1.39 & .97 \\
\hline TIQ36 & High & 29 & 2.39 & .70 \\
\hline & Low & 21 & 2.37 & 1.04 \\
\hline
\end{tabular}

An independent samples T-Test was run for the two groups for the student responsibility and freedom construct.

The results of the independent samples T-Test in table 14 show that, the 2 groups were significantly different in item 31 (We can decide some things in this teacher's class.) $(\mathrm{P}=.00, \mathrm{~T}=2.75)$, and item 35 (This teacher give us a lot of free time in class.) $(\mathrm{P}=.00, \mathrm{~T}=3.17)$. In these items teachers in lower group outperformed the higher one. 
JRCIET

Vol. 7, No. 4

Dctober 2021

Table 14

Independent Samples T-Test for Student responsibility and freedom

Construct

\begin{tabular}{|c|c|c|c|c|c|c|}
\hline & & & & & $\begin{array}{c}95 \% \\
\text { Confiden } \\
\text { ce } \\
\text { Interval }\end{array}$ & \\
\hline & & $\begin{array}{c}\text { Mean } \\
\text { Differen } \\
\text { ce }\end{array}$ & $\mathbf{T}$ & $\begin{array}{l}\text { Sig. (2- } \\
\text { tailed) }\end{array}$ & Lower & $\begin{array}{l}\text { Uppe } \\
\mathbf{r}\end{array}$ \\
\hline \multirow[t]{2}{*}{ TIQ31 } & $\begin{array}{c}\text { Equal variances } \\
\text { assumed }\end{array}$ & .60 & 2.75 & .00 & .16 & 1.04 \\
\hline & $\begin{array}{l}\text { Equal variances } \\
\text { not assumed }\end{array}$ & .60 & 2.64 & .01 & .14 & 1.06 \\
\hline \multirow[t]{2}{*}{ TIQ32 } & $\begin{array}{c}\text { Equal variances } \\
\text { assumed }\end{array}$ & -.30 & -1.12 & .26 & -.84 & .23 \\
\hline & $\begin{array}{l}\text { Equal variances } \\
\text { not assumed }\end{array}$ & -.30 & -1.15 & .25 & -.83 & .22 \\
\hline \multirow[t]{2}{*}{ TIQ33 } & $\begin{array}{c}\text { Equal variances } \\
\text { assumed }\end{array}$ & .10 & .40 & .68 & -.41 & .62 \\
\hline & $\begin{array}{l}\text { Equal variances } \\
\text { not assumed }\end{array}$ & .10 & .42 & .67 & -.38 & .59 \\
\hline \multirow[t]{2}{*}{ TIQ34 } & $\begin{array}{c}\text { Equal variances } \\
\text { assumed }\end{array}$ & -.14 & -.47 & .63 & -.72 & .44 \\
\hline & $\begin{array}{l}\text { Equal variances } \\
\text { not assumed }\end{array}$ & -.14 & -.50 & .61 & -.70 & .42 \\
\hline \multirow[t]{2}{*}{ TIQ35 } & $\begin{array}{c}\text { Equal variances } \\
\text { assumed }\end{array}$ & .76 & 3.17 & .00 & .28 & 1.25 \\
\hline & $\begin{array}{c}\text { Equal variances } \\
\text { not assumed }\end{array}$ & .76 & 3.03 & .00 & .25 & 1.28 \\
\hline \multirow[t]{2}{*}{ TIQ36 } & $\begin{array}{c}\text { Equal variances } \\
\text { assumed }\end{array}$ & -.02 & -.08 & .93 & -.51 & .47 \\
\hline & $\begin{array}{l}\text { Equal variances } \\
\text { not assumed }\end{array}$ & -.02 & -.08 & .93 & -.55 & .51 \\
\hline
\end{tabular}

In Dissatisfied construct the results in table 15 show that, the highest mean score was for item 40 (This teacher thinks that we can't do things well.) (Mean High= 2.89, $\mathrm{SD}=.55$ ), and the lowest score was for item 39 (This teacher puts us down) (Mean High $=1.00, \mathrm{SD}=.89$ ). It is 
illustrated that in both groups item 39 (This teacher puts us down.) received the lowest score while item 40 (This teacher thinks that we can't do things well.) the highest.

Table 15

Group Statistics for Dissatisfied Construct

\begin{tabular}{|c|c|c|c|c|}
\hline & Group & N & Mean & Std. Deviation \\
\hline TIQ37 & High & 29 & 2.62 & 1.12 \\
\hline TIQ38 & High & 29 & 2.17 & .89 \\
\hline & Low & 21 & 1.87 & .77 \\
\hline TIQ39 & High & 29 & 1.00 & .89 \\
\hline & Low & 21 & 1.40 & .80 \\
\hline TIQ40 & High & 29 & 2.89 & .55 \\
\hline & Low & 21 & 2.51 & .53 \\
\hline TIQ41 & High & 29 & 2.87 & .64 \\
\hline & Low & 21 & 2.71 & .56 \\
\hline TIQ42 & High & 29 & 1.76 & .91 \\
\hline & Low & 21 & 1.91 & .67 \\
\hline
\end{tabular}

An independent samples T-Test was run for the two groups for dissatisfied construct. The results of the independent samples T-Test in table 16 show that, the 2 groups were significantly different in item 40 (This teacher thinks that we can't do things well. $)(\mathrm{P}=.01, \mathrm{~T}=-2.43)$. 
Table 16

Independent Samples T-Test for Dissatisfied Construct

\begin{tabular}{|c|c|c|c|c|c|c|}
\hline & & & & & $\begin{array}{c}95 \% \\
\text { Confiden } \\
\text { ce } \\
\text { Interval }\end{array}$ & \\
\hline & & $\begin{array}{c}\text { Mean } \\
\text { Difference }\end{array}$ & $\mathbf{T}$ & $\begin{array}{l}\text { Sig. (2- } \\
\text { tailed) }\end{array}$ & Lower & Upper \\
\hline \multirow[t]{2}{*}{ TIQ37 } & $\begin{array}{c}\text { Equal variances } \\
\text { assumed }\end{array}$ & .00 & .00 & 1.00 & -.57 & .57 \\
\hline & $\begin{array}{l}\text { Equal variances } \\
\text { not assumed }\end{array}$ & .00 & .00 & 1.00 & -.53 & .53 \\
\hline \multirow[t]{2}{*}{ TIQ38 } & $\begin{array}{c}\text { Equal variances } \\
\text { assumed }\end{array}$ & -.30 & -1.24 & .21 & -.79 & .18 \\
\hline & $\begin{array}{c}\text { Equal variances } \\
\text { not assumed }\end{array}$ & -.30 & -1.27 & .20 & -.78 & .17 \\
\hline \multirow[t]{2}{*}{ TIQ39 } & $\begin{array}{c}\text { Equal variances } \\
\text { assumed }\end{array}$ & .40 & 1.62 & .11 & -.09 & .89 \\
\hline & $\begin{array}{c}\text { Equal variances } \\
\text { not assumed }\end{array}$ & .40 & 1.65 & .10 & -.08 & .88 \\
\hline \multirow[t]{2}{*}{ TIQ40 } & $\begin{array}{c}\text { Equal variances } \\
\text { assumed }\end{array}$ & -.38 & -2.43 & .01 & -.69 & -.06 \\
\hline & $\begin{array}{c}\text { Equal variances } \\
\text { not assumed }\end{array}$ & -.38 & -2.45 & .01 & -.69 & -.06 \\
\hline \multirow[t]{2}{*}{ TIQ41 } & $\begin{array}{c}\text { Equal variances } \\
\text { assumed }\end{array}$ & -.15 & -.88 & .37 & -.50 & .19 \\
\hline & $\begin{array}{c}\text { Equal variances } \\
\text { not assumed }\end{array}$ & -.15 & -.90 & .36 & -.50 & .19 \\
\hline \multirow[t]{2}{*}{ TIQ42 } & $\begin{array}{c}\text { Equal variances } \\
\text { assumed }\end{array}$ & .15 & .63 & .52 & -.32 & .62 \\
\hline & $\begin{array}{c}\text { Equal variances } \\
\text { not assumed }\end{array}$ & .15 & .66 & .50 & -.30 & .60 \\
\hline
\end{tabular}


In Strict construct results in table 17 show that, the highest mean score was for item 45 (This teacher's tests are hard.) (Mean High= 3.38, $\mathrm{SD}=.53$ ), and the lowest score was for item 48 (This teacher's standards are very high.) (Mean High $=.71, \mathrm{SD}=.86$ ).

Table 17

Group Statistics for Strict Construct

\begin{tabular}{|c|c|c|c|c|}
\hline & Group & N & Mean & Std. Deviation \\
\hline TIQ43 & High & 29 & 1.03 & .93 \\
\hline TIQ44 & How & 21 & 1.35 & .95 \\
\hline & Low & 21 & 2.46 & .62 \\
\hline TIQ45 & High & 29 & 3.38 & .53 \\
\hline & Low & 21 & 2.68 & .67 \\
\hline TIQ46 & High & 29 & 2.67 & .87 \\
\hline & Low & 21 & 2.52 & .60 \\
\hline TIQ47 & High & 29 & .73 & .76 \\
\hline & Low & 21 & 1.29 & 1.09 \\
\hline TIQ48 & High & 29 & .71 & .86 \\
\hline & Low & 21 & 1.14 & 1.18 \\
\hline & & & & \\
\hline
\end{tabular}

The results of the independent samples T-Test in table 18 show that, the 2 groups were significantly different in item 45 (This teacher's tests are hard.) $(\mathrm{P}=.00, \mathrm{~T}=-4.08)$, and item 47 (We are afraid of this teacher.) ( $\mathrm{P}=.04$, $\mathrm{T}=2.10)$. 
Table 18

Independent Samples T-Test for Strict Construct

\begin{tabular}{|c|c|c|c|c|c|c|}
\hline & \multirow{3}{*}{$\begin{array}{c}\begin{array}{c}\text { Mean } \\
\text { Differenc } \\
\text { e }\end{array} \\
.32\end{array}$} & \multirow[b]{2}{*}{$\mathbf{T}$} & \multirow{2}{*}{$\begin{array}{l}\text { Sig. (2- } \\
\text { tailed) }\end{array}$} & \multicolumn{2}{|c|}{$\begin{array}{c}\text { 95\% Confidence } \\
\text { Interval }\end{array}$} \\
\hline & & & & & Lower & Upper \\
\hline \multirow[t]{2}{*}{ TIQ43 } & $\begin{array}{c}\text { Equal variances } \\
\text { assumed }\end{array}$ & & 1.20 & .23 & -.21 & .86 \\
\hline & $\begin{array}{c}\text { Equal variances } \\
\text { not assumed }\end{array}$ & .32 & 1.20 & .23 & -.22 & .87 \\
\hline \multirow[t]{2}{*}{ TIQ44 } & $\begin{array}{c}\text { Equal variances } \\
\text { assumed }\end{array}$ & -.10 & -.52 & .60 & -.52 & .30 \\
\hline & $\begin{array}{l}\text { Equal variances } \\
\text { not assumed }\end{array}$ & -.10 & -.54 & .58 & -.50 & .29 \\
\hline \multirow[t]{2}{*}{ TIQ45 } & $\begin{array}{c}\text { Equal variances } \\
\text { assumed }\end{array}$ & -.69 & -4.08 & .00 & -1.04 & -.35 \\
\hline & $\begin{array}{c}\text { Equal variances } \\
\text { not assumed }\end{array}$ & -.69 & -3.93 & .00 & -1.05 & -.33 \\
\hline \multirow[t]{2}{*}{ TIQ46 } & $\begin{array}{c}\text { Equal variances } \\
\text { assumed }\end{array}$ & -.14 & -.67 & .50 & -.59 & .29 \\
\hline & $\begin{array}{c}\text { Equal variances } \\
\text { not assumed }\end{array}$ & -.14 & -.71 & .47 & -.56 & .26 \\
\hline \multirow[t]{2}{*}{ TIQ47 } & $\begin{array}{c}\text { Equal variances } \\
\text { assumed }\end{array}$ & .55 & 2.10 & .04 & .02 & 1.08 \\
\hline & $\begin{array}{c}\text { Equal variances } \\
\text { not assumed }\end{array}$ & .55 & 1.99 & .05 & -.01 & 1.11 \\
\hline \multirow[t]{2}{*}{ TIQ48 } & $\begin{array}{c}\text { Equal variances } \\
\text { assumed }\end{array}$ & .42 & 1.48 & .14 & -.15 & 1.01 \\
\hline & $\begin{array}{c}\text { Equal variances } \\
\text { not assumed }\end{array}$ & .42 & 1.41 & .16 & -.18 & 1.04 \\
\hline
\end{tabular}

In "Development" construct of Teaching Performance, the results in table 19 show that, the highest mean score was for item $15(\mathrm{He} / \mathrm{she}$ facilitates student-student and student-professor interaction.) (Mean High $=4.29, \mathrm{SD}=$ $.50)$, and the lowest score was for item 9 (He/she promotes individual work.) (Mean Low $=2.65, \mathrm{SD}=.71$ ). 
Table 19

Group Statistics for Development Construct

\begin{tabular}{|c|c|c|c|c|}
\hline \multirow{3}{*}{ TPQ1 } & Group & $\mathbf{N}$ & Mean & Std. Deviation \\
\hline & High & 29 & 3.90 & .77 \\
\hline & Low & 21 & 3.74 & .66 \\
\hline \multirow{2}{*}{ TPQ2 } & High & 29 & 3.94 & .85 \\
\hline & Low & 21 & 3.67 & .56 \\
\hline \multirow[t]{2}{*}{ TPQ3 } & High & 29 & 3.95 & .62 \\
\hline & Low & 21 & 3.54 & .41 \\
\hline \multirow[t]{2}{*}{ TPQ7 } & High & 29 & 4.08 & .58 \\
\hline & Low & 21 & 4.04 & .37 \\
\hline \multirow{2}{*}{ TPQ8 } & High & 29 & 4.10 & .60 \\
\hline & Low & 21 & 3.98 & .56 \\
\hline \multirow[t]{2}{*}{ TPQ9 } & High & 29 & 3.02 & .69 \\
\hline & Low & 21 & 2.65 & .71 \\
\hline \multirow[t]{2}{*}{ TPQ10 } & High & 29 & 4.08 & .92 \\
\hline & Low & 21 & 3.94 & .58 \\
\hline \multirow[t]{2}{*}{ TPQ11 } & High & 29 & 3.95 & .58 \\
\hline & Low & 21 & 3.23 & .45 \\
\hline \multirow{2}{*}{ TPQ12 } & High & 29 & 3.94 & .61 \\
\hline & Low & 21 & 3.67 & .40 \\
\hline \multirow[t]{2}{*}{ TPQ13 } & High & 29 & 4.21 & .56 \\
\hline & Low & 21 & 3.94 & .60 \\
\hline \multirow[t]{2}{*}{ TPQ15 } & High & 29 & 4.29 & .50 \\
\hline & Low & 21 & 3.90 & .66 \\
\hline \multirow[t]{2}{*}{ TPQ16 } & High & 29 & 4.26 & .77 \\
\hline & Low & 21 & 3.88 & .66 \\
\hline \multirow{2}{*}{ TPQ17 } & High & 29 & 3.95 & .66 \\
\hline & Low & 21 & 3.68 & .58 \\
\hline \multirow[t]{2}{*}{ TPQ18 } & High & 29 & 4.00 & .71 \\
\hline & Low & 21 & 3.56 & .83 \\
\hline \multirow[t]{2}{*}{ TPQ19 } & High & 29 & 3.93 & .61 \\
\hline & Low & 21 & 3.63 & .50 \\
\hline \multirow[t]{2}{*}{ TPQ23 } & High & 29 & 3.83 & .59 \\
\hline & Low & 21 & 3.46 & .62 \\
\hline \multirow[t]{2}{*}{ TPQ26 } & High & 29 & 4.26 & .64 \\
\hline & Low & 21 & 3.93 & .67 \\
\hline
\end{tabular}

The results of the independent samples T-Test in table 20 show that, the 2 groups were significantly different in item $3 \mathrm{(He} / \mathrm{she}$ allows the students to organize and distribute part of the assignment to be performed in the course.) $(\mathrm{P}=.01, \mathrm{~T}=-2.63)$, item $11(\mathrm{He} / \mathrm{she}$ relates the teachings to the professional environment.) $(\mathrm{P}=.00, \mathrm{~T}=-$ 4.73), item $15(\mathrm{He} / \mathrm{she}$ facilitates student-student and student-professor interaction.) $(\mathrm{P}=.02, \mathrm{~T}=-2.35)$, and item $23(\mathrm{He} / \mathrm{she}$ interweaves the content of the subject matter with other courses. $)(\mathrm{P}=.03, \mathrm{~T}=-2.14)$. 
Table 20

\section{Independent Samples T-Test for Development Construct}

\begin{tabular}{|c|c|c|c|c|c|c|}
\hline & \multirow{2}{*}{$\begin{array}{c}\text { Mean } \\
\text { Difference }\end{array}$} & \multirow[b]{2}{*}{$\mathbf{T}$} & \multirow{2}{*}{$\begin{array}{l}\text { Sig. (2- } \\
\text { tailed) }\end{array}$} & \multicolumn{2}{|c|}{$\begin{array}{c}95 \% \text { Confidence } \\
\text { Interval } \\
\end{array}$} \\
\hline & & & & & Lower & Upper \\
\hline \multirow[t]{2}{*}{ TPQ1 } & Equal variances assumed & -.15 & -.76 & .45 & -.57 & .26 \\
\hline & Equal variances not assumed & -.15 & -.77 & .44 & -.56 & .25 \\
\hline \multirow[t]{2}{*}{ TPQ2 } & Equal variances assumed & -.26 & -1.23 & .22 & -.69 & .16 \\
\hline & Equal variances not assumed & -.26 & -1.32 & .19 & -.67 & .13 \\
\hline \multirow[t]{2}{*}{ TPQ3 } & Equal variances assumed & -.41 & -2.63 & .01 & -.72 & -.09 \\
\hline & Equal variances not assumed & -.41 & -2.81 & .00 & -.70 & -.11 \\
\hline \multirow[t]{2}{*}{ TPQ7 } & Equal variances assumed & -.03 & -.23 & .81 & -.32 & .25 \\
\hline & Equal variances not assumed & -.03 & -.25 & .80 & -.30 & .23 \\
\hline \multirow[t]{2}{*}{ TPQ8 } & Equal variances assumed & -.12 & -.75 & .45 & -.46 & .21 \\
\hline & Equal variances not assumed & -.12 & -.76 & .45 & -.46 & .20 \\
\hline \multirow[t]{2}{*}{ TPQ9 } & Equal variances assumed & -.37 & -1.85 & .07 & -.77 & .03 \\
\hline & Equal variances not assumed & -.37 & -1.84 & .07 & -.78 & .03 \\
\hline \multirow[t]{2}{*}{ TPQ10 } & Equal variances assumed & -.14 & -.63 & .52 & -.60 & .31 \\
\hline & Equal variances not assumed & -.14 & -.68 & .49 & -.57 & .28 \\
\hline \multirow[t]{2}{*}{ TPQ11 } & Equal variances assumed & -.72 & -4.73 & .00 & -1.03 & -.41 \\
\hline & Equal variances not assumed & -.72 & -4.92 & .00 & -1.01 & -.42 \\
\hline \multirow[t]{2}{*}{ TPQ12 } & Equal variances assumed & -.26 & -1.73 & .08 & -.57 & .04 \\
\hline & Equal variances not assumed & -.26 & -1.85 & .07 & -.55 & .02 \\
\hline \multirow[t]{2}{*}{ TPQ13 } & Equal variances assumed & -.26 & -1.59 & .11 & -.59 & .06 \\
\hline & Equal variances not assumed & -.26 & -1.57 & .12 & -.60 & .07 \\
\hline \multirow[t]{2}{*}{ TPQ15 } & Equal variances assumed & -.38 & -2.35 & .02 & -.71 & -.05 \\
\hline & Equal variances not assumed & -.38 & -2.25 & .03 & -.73 & -.03 \\
\hline \multirow[t]{2}{*}{ TPQ16 } & Equal variances assumed & -.37 & -1.80 & .07 & -.80 & .04 \\
\hline & Equal variances not assumed & -.37 & -1.85 & .07 & -.79 & .03 \\
\hline \multirow[t]{2}{*}{ TPQ17 } & Equal variances assumed & -.26 & -1.45 & .15 & -.63 & .10 \\
\hline & Equal variances not assumed & -.26 & -1.48 & .14 & -.62 & .09 \\
\hline \multirow[t]{2}{*}{ TPQ18 } & Equal variances assumed & -.43 & -1.97 & .05 & -.87 & .00 \\
\hline & Equal variances not assumed &.- .43 & -1.92 & .06 & -.89 & .02 \\
\hline \multirow[t]{2}{*}{ TPQ19 } & Equal variances assumed & -.29 & -1.80 & .07 & -.62 & .03 \\
\hline & Equal variances not assumed & -.29 & -1.85 & .07 & -.61 & .02 \\
\hline \multirow[t]{2}{*}{ TPQ23 } & Equal variances assumed & -.37 & -2.14 & .03 & -.72 & -.02 \\
\hline & Equal variances not assumed & -.37 & -2.12 & .03 & -.72 & -.01 \\
\hline \multirow[t]{2}{*}{ TPQ26 } & Equal variances assumed & -.32 & -1.72 & .09 & -.70 & .05 \\
\hline & Equal variances not assumed & -.32 & -1.71 & .09 & -.70 & .05 \\
\hline
\end{tabular}


In "Planning" construct of Teaching Performance, the results in table 21 show that, the highest mean score was for item $22(\mathrm{He} / \mathrm{she}$ has a good command of the contents of the course.) (Mean High= 3.95, $\mathrm{SD}=.61$ ), and the lowest score was for item $20(\mathrm{He} / \mathrm{she}$ designs and relates the classroom content to the lab content.) (Mean Low $=3.08$, $\mathrm{SD}=.57)$.

Table 21

Group Statistics for Planning Construct

\begin{tabular}{|c|c|c|c|c|}
\hline & Group & $\mathbf{N}$ & Mean & Std. Deviation \\
\hline \multirow{2}{*}{ TPQ4 } & High & 29 & $\mathbf{3 . 8 9}$ & $\mathbf{. 9 0}$ \\
\cline { 2 - 5 } & Low & 21 & $\mathbf{3 . 8 2}$ & .47 \\
\hline \multirow{2}{*}{ TPQ20 } & High & 29 & $\mathbf{3 . 4 9}$ & .66 \\
\cline { 2 - 5 } & Low & 21 & $\mathbf{3 . 0 8}$ & .57 \\
\hline \multirow{2}{*}{ TPQ21 } & High & 29 & $\mathbf{3 . 8 4}$ & $\mathbf{. 6 7}$ \\
\cline { 2 - 5 } & Low & 21 & $\mathbf{3 . 5 1}$ & .75 \\
\hline \multirow{2}{*}{ TPQ22 } & High & 29 & $\mathbf{3 . 9 5}$ & $\mathbf{. 6 1}$ \\
\cline { 2 - 5 } & Low & 21 & $\mathbf{3 . 6 4}$ & .53 \\
\hline
\end{tabular}

The results of the independent samples T-Test in table 22 show that, the 2 groups were significantly different in item $20(\mathrm{He} / \mathrm{she}$ designs and relates the classroom content to the lab content.) $(\mathrm{P}=.02, \mathrm{~T}=-2.25)$.

Table 22

Independent Samples T-Test for Planning Construct

\begin{tabular}{|c|c|c|c|c|c|c|}
\hline & \multirow{2}{*}{$\begin{array}{c}\text { Mean } \\
\text { Difference }\end{array}$} & \multirow[b]{2}{*}{$\mathbf{T}$} & \multirow{2}{*}{$\begin{array}{l}\text { Sig. (2- } \\
\text { tailed) }\end{array}$} & \multicolumn{2}{|c|}{$\begin{array}{l}\text { 95\% Confidence } \\
\text { Interval } \\
\end{array}$} \\
\hline & & & & & Lower & Upper \\
\hline \multirow[t]{2}{*}{ TPQ4 } & Equal variances assumed & \begin{tabular}{|c|}
.07 \\
\end{tabular} & -.35 & .72 & -.51 & .36 \\
\hline & $\begin{array}{c}\begin{array}{c}\text { Equal variances not } \\
\text { assumed }\end{array} \\
\end{array}$ & -.07 & -.38 & .70 & -.47 & .32 \\
\hline \multirow[t]{2}{*}{ TPQ20 } & Equal variances assumed & -.40 & -2.25 & .02 & -.77 & -.04 \\
\hline & $\begin{array}{c}\begin{array}{c}\text { Equal variances not } \\
\text { assumed }\end{array} \\
\end{array}$ & -.40 & -2.30 & .02 & -.76 & -.05 \\
\hline \multirow[t]{2}{*}{ TPQ21 } & Equal variances assumed & -.33 & -1.62 & .11 & -.74 & .07 \\
\hline & $\begin{array}{c}\begin{array}{c}\text { Equal variances not } \\
\text { assumed }\end{array} \\
\end{array}$ & -.33 & -1.60 & .11 & -.75 & .08 \\
\hline \multirow[t]{2}{*}{ TPQ22 } & Equal variances assumed &.- .30 & -1.85 & .07 & -.64 & .02 \\
\hline & \begin{tabular}{|c|}
$\begin{array}{c}\text { Equal variances not } \\
\text { assumed }\end{array}$ \\
\end{tabular} & -.30 & -1.89 & .06 & -.63 & .01 \\
\hline
\end{tabular}


In the "Result" construct (table 23) it is shown that, the highest mean score was for item 6 (He/she provides me with scientific information that allows me to gain a better and deeper understanding of the subject matter.) (Mean High= 4.15, SD= .66), and the lowest score was for item 14 (He/she fosters research and a critical spirit in students.) (Mean Low $=3.37, \mathrm{SD}=.62$ ).

Table 23

Group Statistics for Result Construct

\begin{tabular}{|c|c|c|c|c|}
\hline & Group & N & Mean & Std. Deviation \\
\hline TPQ5 & High & 29 & 3.94 & .56 \\
\cline { 2 - 5 } & Low & 21 & 3.65 & .55 \\
\hline \multirow{2}{*}{ TPQ6 } & High & 29 & 4.15 & .66 \\
\cline { 2 - 5 } & Low & 21 & 3.71 & .62 \\
\hline TPQ14 & High & 29 & 3.79 & .77 \\
\cline { 2 - 5 } & Low & 21 & 3.37 & .62 \\
\hline TPQ24 & High & 29 & 3.85 & .65 \\
\cline { 2 - 5 } & Low & 21 & 3.58 & .68 \\
\hline TPQ25 & High & 29 & 3.93 & .83 \\
\cline { 2 - 5 } & Low & 21 & 3.56 & .84 \\
\hline TPQ27 & High & 29 & 3.70 & .70 \\
\cline { 2 - 5 } & Low & 21 & 3.44 & .48 \\
\hline TPQ28 & High & 29 & 3.73 & .64 \\
\cline { 2 - 5 } & Low & 21 & 3.43 & .64 \\
\hline
\end{tabular}

The results of the independent samples T-Test in table 24 show that, the 2 groups were significantly different in item $6(\mathrm{He} / \mathrm{she}$ provides me with scientific information that allows me to gain a better and deeper understanding of the subject matter.) ( $\mathrm{P}=.02, \mathrm{~T}=-2.38)$ and item $14(\mathrm{He} / \mathrm{she}$ fosters research and a critical spirit in students.) ( $\mathrm{P}=.04$, $\mathrm{T}=-2.03$ ). 
Table 24

Independent Samples T-Test for Result Construct

\begin{tabular}{|c|c|c|c|c|c|c|}
\hline & \multirow{2}{*}{$\begin{array}{c}\text { Mean } \\
\text { Difference }\end{array}$} & \multirow[b]{2}{*}{$\mathbf{T}$} & \multirow{2}{*}{$\begin{array}{l}\text { Sig. (2- } \\
\text { tailed) }\end{array}$} & \multicolumn{2}{|c|}{$\begin{array}{c}\text { 95\% Confidence } \\
\text { Interval }\end{array}$} \\
\hline & & & & & Lower & Upper \\
\hline \multirow[t]{2}{*}{ TPQ5 } & Equal variances assumed & -.29 & -1.82 & .07 & -.61 & .03 \\
\hline & $\begin{array}{c}\text { Equal variances not } \\
\text { assumed }\end{array}$ & -.29 & -1.83 & .07 & -.61 & .02 \\
\hline \multirow[t]{2}{*}{ TPQ6 } & Equal variances assumed & -.44 & -2.38 & .02 & -.81 & -.06 \\
\hline & $\begin{array}{c}\text { Equal variances not } \\
\text { assumed }\end{array}$ & -.44 & -2.41 & .02 & -.80 & -.07 \\
\hline \multirow[t]{2}{*}{ TPQ14 } & Equal variances assumed & -.41 & -2.03 & .04 & -.82 & -.00 \\
\hline & $\begin{array}{c}\text { Equal variances not } \\
\text { assumed }\end{array}$ & -.41 & -2.10 & .04 & -.81 & -.01 \\
\hline \multirow[t]{2}{*}{ TPQ24 } & Equal variances assumed & -.27 & -1.40 & .16 & -.65 & .11 \\
\hline & \begin{tabular}{|c|}
$\begin{array}{c}\text { Equal variances not } \\
\text { assumed }\end{array}$ \\
\end{tabular} & -.27 & -1.40 & .16 & -.65 & .11 \\
\hline \multirow[t]{2}{*}{ TPQ25 } & Equal variances assumed & -.37 & -1.57 & .12 & -.86 & .10 \\
\hline & \begin{tabular}{|c|}
$\begin{array}{c}\text { Equal variances not } \\
\text { assumed }\end{array}$ \\
\end{tabular} & -.37 & -1.57 & .12 & -.86 & .10 \\
\hline \multirow[t]{2}{*}{ TPQ27 } & Equal variances assumed & -.26 & -1.46 & .15 & -.61 & .09 \\
\hline & \begin{tabular}{|c|}
$\begin{array}{c}\text { Equal variances not } \\
\text { assumed }\end{array}$ \\
\end{tabular} & -.26 & -1.54 & .12 & -.59 & .07 \\
\hline \multirow[t]{2}{*}{ TPQ28 } & Equal variances assumed & -.29 & -1.61 & .11 & -.66 & .07 \\
\hline & \begin{tabular}{|c|}
$\begin{array}{c}\text { Equal variances not } \\
\text { assumed }\end{array}$ \\
\end{tabular} & -.29 & -1.61 & .11 & -.67 & .07 \\
\hline
\end{tabular}

Results showed that in Teacher Interaction, the 2 groups were significantly different in 21 items out of 48 ones $(2,5,6,9,10,13,14,15,17,18,19,20,21,23,24$, $25,27,29,31,35,40,45,47)$.

In Teaching Performance, the 2 groups were significantly different in 6 items out of 28 ones $(6,11,14$, $15,20,23$,$) and in all 28$ items teachers in higher group outperformed the lower one.

\section{Discussion}

Teaching performance is one of the most important factors in successful language teaching. In order to be a 
successful teacher, it is better to pay more attention to the development part of the teaching process rather than planning and result. In order to have better development, the teacher should facilitate student-student and studentprofessor interaction, attend clearly to the questions asked in the class, and interact satisfactorily with the students. Related studies such as (Faravani \& Ataei, 2015a; Faravani \& Ataei, 2015b; Rohmah, 2017) proved that, it is important for the teacher to build interactive and communicative teaching-learning activities involving more interaction and participation among the students. Also, the classroom interaction is an effective pedagogical tool for increasing learner's language use and advancing classroom participation, which in turn improves student's speaking skill (Warda, 2015). Furthermore, interaction is essential for the teacher-student relationship and a positive relationship between the students and their teachers positively impacts the students' interest and motivation in school, which contributes to the improvement of the learning process (Soares, 2015).

For the planning part, the teacher should have a good command of the contents of the course, provide clear information about objectives, bibliography, tutorials, content, and assessment methods in the subject's curriculum and efficiently y incorporate and employ Information, Communication, and Technology (ICT). The related studies stated that ICT has a great effectiveness for both teachers and students. Teachers' preparation with ICT tools and facilities is one the key element in the success of technology-based teaching and learning (Ghavifekr, Athirah, \& Rosdy, 2015). Also, the impact of ICT for teaching and learning process is proved because it 
facilitates teaching and learning process, create a friendly learning environment, and help learners develop creative thinking and self-confidence (Nwigbo \& Madhu, 2016).

As for Teacher interaction, uncertain behaviour is very necessary in the case of teacher interaction. In helpful and friendly behaviour, the teacher can take a joke and also help students with their work. In related studies, it is proved that a successful teacher must be knowledgeable, responsible, enthusiastic, patient and kind and acts friendly with students, providing support for them and help them with learning, and encouraging professional development (Huang, 2010). However, Humor is considered as an important factor to decrease the anxiety and boredom in English language classes. It makes English language class more effective and interesting and creates a friendly learning environment that helps the students to perform better (Abdullah \& Akhter, 2015). Also, it is proved that humor has a special impact on the classroom climate and is considered as an effective tool for teaching. It can create positive relationships between teachers and students, decrease the stress level of the classroom, facilitate learning and develop creativity of students (Jeder, 2014). Also, humor considered as an important factor in social life and when used in the English language classroom by teachers, it could facilitate the students' learning and make teaching more effective (Nayyar \& Zeeshan, 2017).

In leadership behaviour, to be a successful teacher, the teacher should be a good leader and he/she should explain things clearly. Leadership is an essential feature for the teacher's effectiveness and empowerment (Emmanouil, Osia \& Paraskevi-Ioanna, 2014). Besides, leadership in 
teaching works towards both improving learning and teaching at the same time. High quality leaders may achieve this impact on the teaching/learning process through establishing clear directions for everyone to follow, providing teachers with training that will enhance their teaching techniques as well as finding suitable conditions in order to help both teaching and learning (Kawar, 2012).

In dissatisfied behaviour, the teacher should think that students can do things well. In strict behaviour the successful teacher's tests are not hard and he/she is not very severe when marking papers. In admonishing behaviour, the teacher is too quick to correct students when they break a rule. In student responsibility and freedom behaviour, the teachers are lenient and let students fool around in class.

\section{Conclusions}

Based on the findings of this study in teaching performance, "development" and "planning" were more important than result. Thus, teachers need to consider these constructs in the teaching process. Another study also indicated that planning and development are fundamental constructs in teaching (Moreno-Murcia et al., 2015). By planning, a teacher is able to manage time, effort and resources efficiently. Besides, it provides the teachers many ways such as variation of activities, methods and materials to keep the teaching process not monotonous and redundant. Furthermore, it can help teachers to achieve the goals and objectives appropriately as well as help them have great self-confidence and get rid of problems (Jamali Nesari \& Heidari, 2014). 
Furthermore, according to the results of teacher interaction, uncertain behaviour as well as helpful and friendly and also leadership behaviour were more important than the other constructs. Thus, teachers should consider these constructs more in their teaching process. The importance of leadership and helpful and friendly behaviour is highlighted in the literature. Moreover, in another study helpful and understanding behaviour was considered as key factors in successful teaching (Sun, Mainhard, \& Wubbels, 2017).

\section{References}

- Adaba, H. W. (2017). Exploring the Practice of Teacher-Student Classroom Interaction in EFL to Develop the Learners' Speaking Skills in Tullu Sangota Primary School Grade Eight Students in Focus. Arts and Social Sciences Journal 8(4).

- Choudhury, Sh. (2005). Interaction in second language classrooms. BRAC University Journal, 2(1), 77-82.

- Dubey, N. (2016). Role of Teacher in Modern Society. Retrieved rom http:// www.dailyexcelsior.com

- Elliott, K. (2015). Teacher Performance Appraisal: More about Performance or Development. Australian Journal of Teacher Education, 40(9).

- Emmanouil, K., Osia, A., \& Paraskevi-Ioanna, L. (2014). The Impact of Leadership on Teachers' Effectiveness. International Journal of Humanities and Social Science, 4(7).

- Eschenmann, K. K. (2015). Student Perceptions of Teaching Style in the Health Occupations Classroom. Journal of Health Occupations Education, 6 (1).

- Faramarz Zadeh, R. (2016). EFL Learners' Attitudes and Perceptions about an Effective English Language Teacher. Journal of Applied Linguistics and Language Research, 3 (2). Retrieved from $w w w . j a l l r . c o m$

- Faravani, A. (2021). رو انشناسى و تربيت آموزشى. Ghom: Farzanegane Danesh Publication. 
- Faravani, A., \& Atai, M. R. (2015a). Multiple Intelligences, Dialogic-Based Portfolio Assessment, and the Enhancement of Higher Order Thinking. Journal of Teaching Language Skills, 33(4), 19-44.

- Faravani, A., \& Atai, M. R. (2015b). Portfolio Assessment and the Enhancement of Higher Order Thinking through Multiple Intelligence and Dialogic Feedback. Issues in Language Teaching, 4(1), 25-1.

- Fernández, D.M., \& Pérez Cañado, L. (2001). Exploring the student's motivation in the EFL class. Present and Future Trends in TEFL, 321-364.

- Ghavifekr, S. \& Rosdy, W.A.W. (2015). Teaching and learning with technology: Effectiveness of ICT integration in schools. International Journal of Research in Education and Science (IJRES), 1(2), 175-191.

- Huang, Z. (2010). What Makes a Successful EFL Teacher in China? A Case Study of an English Language Teacher at Nanjing University of Chinese Medicine. English Language Teaching, 3(3).

- Jamali Nesari, A. (2014). The Important Role of Lesson Plan on Educational Achievement of Iranian EFL Teachers' Attitudes. International Journal of Foreign Language Teaching \& Research, 3(5), 25-31.

- Jeder, D. (2015). Implications of using Humor in the Classroom. Procedia - Social and Behavioral Sciences, 180, 828833.

- Kaufman, R. (2005). Defining and Delivering Measurable Value: A Mega Thinking and Planning Primer. Performance Improvement Quarterly, 18(3), 6-16.

- Kaufman, R. (2009). Mega Thinking and Planning: An Introduction to Defining and Delivering Individual and Organizational Success. Performance Improvement Quarterly, 22(2). 
- Kawar, T.I. (2012). The Impact of Leadership on Student Learning', International Journal of Business and Social Science, 3(8).

- Moafian, F. \& Pishghadam, R. (2009). Design and validation of the questionnaire on successful English professors' characteristics. Foreign language research, (54), 127-142.

- Montoneri, B. (2016). Evaluation of teaching performance of English courses by applying data envelopment analysis and twophase segmentation. The Third Asian Conference on Education 2011 Official Proceedings, 377-391.

- Moreno-Murcia, J.A., Torregrosa, Y.S., \& Pedreño, N.B. (2015). Questionnaire evaluating teaching competencies in the university environment. Evaluation of teaching competencies in the university. New Approaches in Educational Research, 4(1), 54-61.

- Nayyar, R., \& Zeeshan, M. (2017). MPhil Scholars' Views about the Use of Humor in English Language Classroom in Quetta, Balochistan, Pakistan. International Journal of English Linguistics, 7(2).

- Nwigbo,S., \& Madhu, B.K. (2016). Impact of ICT on the Teaching and Learning Process. IOSR Journal of Mobile Computing \& Application (IOSR-JMCA), 3(2), 01-07.

- Rohmah, I. I. T. (2017). Classroom Interaction in English Language Class for Students of Economics Education. Arab World English Journal (AWEJ), 8 (2).

- Soares dos Reis da Luz, F. (2015). The Relationship between Teachers and Students in the Classroom: Communicative Language Teaching Approach and Cooperative Learning Strategy to Improve Learning. BSU Master's Theses and Projects. Item 22. Retrieved from http://vc.bridgew.edu/theses/22

- Sun, X., Mainhard, T., \& Wubbels, T. (2017). Development and evaluation of a Chinese version of the Questionnaire on Teacher Interaction (QTI). Learning Environ Res.

- Suwandee, A. (1995). Students' Perceptions of University Instructors' Effective Teaching characteristics. SLLT Journal, 5, 622. 
- Warda, S. (2015). The Importance of Classroom Interaction in Improving EFL Student's Speaking Skill. University of Biskra.

- Wubbels, Th., \& Levy, J. (Eds.). (1993). Do you know what you look like: Interpersonal relationships in education. London, England: Falmer Press.

- Zhi-Feng Liu, E., Lin, Ch., Liou, P., Feng, H., \& Hou, H. (2013). An analysis of teacher-student interaction patterns in a robotics course for kindergarten children: a pilot study'. The Turkish Online Journal of Educational Technology, 12(1).

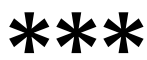

